\title{
Breast cancer in moroccan young women: a retrospective study
}

\author{
Halima Abahssain ${ }^{1 *}$, Issam Lalya ${ }^{2}$, Fatima zahra EL M'Rabet ${ }^{3}$, Nabil Ismailii ${ }^{*}$, Rachid Razine ${ }^{4}$, \\ Mohammed Adnane Tazi ${ }^{5}$, Hind M'rabti ${ }^{1}$, Omar El Mesbahi ${ }^{3}$, Nourddine Benjaafar ${ }^{2}$, \\ Redouane Abouqal ${ }^{4}$, Hassan Errihani ${ }^{1}$
}

\begin{abstract}
Background: Breast cancer is uncommon in young women and induces more aggressive biologic characteristics. Survival in young women has been widely studied in developed countries. Less favorable prognosis and low survival were found.

In Morocco, this study is the first investigation of clinical features, treatment and prognosis associated with breast cancer in young women.
\end{abstract}

Findings: Four hundred and nine women aged 35 years or less were included in this study. All these women were diagnosed as having breast cancer at the National Institute of Oncology in Rabat, Morocco between 2003 and 2007. The relation between clinical and therapeutic characteristics and event-free survival (EFS) and overall survival (OS) were assessed by Cox regression analysis.

The median age of the patients was 32 years. Fifty three patients (13\%) have metastatic disease at diagnosis and 356 patients (87\%) had localised disease. In $57.9 \%$ of the cases, the estrogen receptors status was positive. The median follow-up was 32.2 months. After 3 years the survival rate was $80.6 \%$. In the case of localised disease, OS and EFS at 3 years were $83.2 \%$ and $62.5 \%$, respectively. OS and EFS at 3 years was higher in patients with stage I than patients with stage II and stage III $(p=0.001)$. Positive estrogen receptors was significantly associated to OS and EFS at 3 years compared to negative estrogen receptors $(p=0.001)$. Adjuvant chemotherapy, adjuvant radiotherapy and adjuvant hormone therapy were associated with net benefit in OS and EFS at 3 years. Cox regression analysis showed that negative ER was significantly associated with poorer OS ( $H R=2.42,95 \% \mathrm{Cl}=1.25-4.66$, $\mathrm{p}<0.009)$ and poorer EFS $(\mathrm{HR}=1.73,95 \% \mathrm{Cl}=1.05-2.86, \mathrm{p}=0.03)$. Stage III disease were associated to poorer $\mathrm{EFS}(\mathrm{HR}=5.35,95 \% \mathrm{Cl}=1.60-17.84, \mathrm{p}=0.006)$.

Conclusions: In Morocco, young women with breast cancer had less favorable prognosis. Multivariate analysis showed that negative hormone receptor status was associated with lower EFS and OS. Clinical trials should be launched to improve the survival of these young women with breast cancer.

\section{Background}

Breast cancer in young women is uncommon. Approximately $2 \%$ of patients with breast carcinoma are age $\leq$ 35 years old at the time of diagnosis [1,2]. Information is limited in this category of women. The definition of "young women" varied from 30, 35, 40, 45, or even 50 years [3]. Young patients with breast cancer had more aggressive clinical and biological characteristics, less

\footnotetext{
*Correspondence: abahssainhalima@yahoo.fr; ismailinabil@yahoo.fr 'Department of medical oncology, National Institute of Oncology, Rabat, Morocco

Full list of author information is available at the end of the article
}

favorable outcome, and the disease was more linked to a genetic predisposition compared with the disease in older patients [4-7].

According to the 1998 St Gallen guidelines, age $\leq 35$ was a poor prognostic factor and supported the use of more aggressive systemic therapy, including chemotherapy in all younger patients regardless of other factors [8].

In addition, other particularities must be studied in this category of patients: fertility, menopause induced by treatment, self-image and sexuality.

\section{Biomed Central}


The aim of the present study was to investigate the epidemiological, clinical and treatment characteristics in young patients with breast cancer, and their relationship with event free survival and overall survival.

\section{Patients and methods \\ Clinical data}

The National Institute of Oncology database was used to identify patients with breast cancer aged 35 years or less at the time of diagnosis between 2003 and 2007. We excluded from the study patients who had not follow up after initial diagnosis. The scientific comity of National Institute of Oncology approved the retrospective review of the medical records for the purposes of the current study. Breast carcinoma diagnosis was made by biopsy of the breast tumor. Tumor staging was carried out according to the TNM classification 2002 modified in 2003. Histological tumor grading was performed using the Scarff Bloom and Richardson (SBR) histological system.

Immunohistochemical analysis to determine estrogen (ER) and progesterone receptor (PR) status was performed using standard procedures on 4- $\mu \mathrm{m}$ sections of paraffinembedded tissue specimens stained with the monoclonal antibodies 6F11 and 1A6 for ER and PR, respectively. Nuclear staining $10 \%$ was considered a positive result.

Patients were considered HER2-positive if they had immunohistochemistry (IHC) 3+ by DAKO HercepTest. Fluorescence in situ hybridization was used when IHC was $2+$ and it was considered positive if we have $\geq$ twofold amplification; confirmed by central testing. The hercept test was realized in the institute from 2004.

\section{Treatment}

Patients how had metastatic disease at diagnosis received chemotherapy or hormonotherapy based on the characteristics of the tumors and the aggressiveness of the disease. This patients can receive palliative radiotherapy if indication. Patients with local disease had received corresponding local treatments (surgery plus radiotherapy) and systemic treatments (mainly adjuvant and/or neoadjuvante chemotherapy and endocrine therapy). The main surgical operations included radical mastectomy (Patey type mastectomy) and breast conserving surgery when permitted by tumor size according to the judgment of the multidisciplinary care team.

In our institute, adjuvant chemotherapy is indicated in the case of tumor size greater than or equal to $2 \mathrm{~cm}$, positive nodal status, grade 2 or $3 \mathrm{SBR}$, amplification of HER 2 and age $\leq 35$ years. Neoadjuvante chemotherapy was giving in patients with inflammatory or locally advanced breast cancer. For the patients who did not receive adjuvant chemotherapy, they have received neoadjuvante chemotherapy or they did not return after surgery for personal reasons.

For patients receiving neoadjuvante chemotherapy, a pathologic complete response was defined as no evidence of invasive carcinoma in the breast and the axillary lymph nodes at the time of surgery. Chevalier classification was used to classify histological response to neoadjuvante chemotherapy in the breast [9].

Anthracycline containing regimens were mainly used for adjuvant and/or neoadjuvante chemotherapy (FEC 100 protocol with Fluorouracil $500 \mathrm{mg} / \mathrm{m}^{2}$, Epirubicin $100 \mathrm{mg} / \mathrm{m}^{2} \mathrm{IV}$, Cyclophosphamide $500 \mathrm{mg} / \mathrm{m}^{2}$ and AC60 protocol with Doxorubicin $60 \mathrm{mg} / \mathrm{m}^{2}$, Cyclophosphamide $600 \mathrm{mg} / \mathrm{m}^{2}$ ) while CMF regimen (Cyclophosphamide $600 \mathrm{mg} / \mathrm{m}^{2}$, Metotrexate $40 \mathrm{mg} / \mathrm{m}^{2}$, Fluorouracil: $500 \mathrm{mg} / \mathrm{m}^{2}$ ) was administrated instead in some minor cases. Docetaxel was administered at a dose of $100 \mathrm{mg} / \mathrm{m}^{2}$ when used in monotherapy and at a dose of $75 \mathrm{mg} / \mathrm{m}^{2}$ in combination with anthracyclin. The choice of chemotherapy protocols depended on the availability of products at the time of the indication.

Patients with hormone receptor positive tumor specimens received tamoxifen at a dose of $20 \mathrm{mg}$ daily for 5 years. Adjuvant radiotherapy was indicated the case of tumor size greater than $5 \mathrm{~cm}$, invasion of the pectoral fascia, more than four metastatic axillary lymph nodes, positive surgical margin and breast conservation.

\section{Follow up}

Patients were followed up until January 2010. All patients who are not reviewed in the last consultation were contacted again by telephone. Locoregional recurrence meant the recurrence in ipsilateral mammary glands, chest wall, or regional lymph nodes identified clinically or histologically, while distant metastasis referred to the metastatic carcinoma detected by clinical examination or imaging. Event free survival (EFS) was calculated from the date of surgery or the first course of neoadjuvante chemotherapy to the date of event (loco regional or metastatic relapse, or death) or last follow up. Patients with stage IV disease at diagnosis were excluded from the statistical evaluation of EFS. Overall survival (OS) was calculated from the date of diagnosis (fin needles aspiration, biopsies or radical mastectomy) to the date of death or last follow-up.

\section{Statistical analysis}

SPSS13.0 software was used for statistical analysis. Descriptive of clinical data were expressed in percentage or median or mean \pm SD. Survival was estimated by the Kaplan Meier method, and compared by the log rank test. The relationship between each of the explanatory variables and outcome (EFS and OS) was assessed in 
turn using univariate and multivariate Cox's regression analysis. A p value of $<0.05$ was considered significant.

Consent and statement of ethical approval

As the treatment of each patient was decided by the medical staff of the centre, oral consent was obtained from the subjects and was approved by the institutional review boards of the National Institute of Oncology, Cancer Centre in Rabat. This study was approved by the institutional review boards of National Institute of Oncology, in Rabat.

\section{Results}

\section{Clinical characteristics of all patients}

Four hundred and twenty seven patients aged 35 years or younger were diagnosed with breast cancer between January 2003 and December 2007. Four hundred and nine patients were included. The median follow-up time

Table 1 Clinical characteristics of all Patients

\begin{tabular}{|c|c|c|}
\hline Characteristics & No. of patients & (\%) \\
\hline Nursing & 121 & 59 \\
\hline \multicolumn{3}{|c|}{ Family history of breast carcinoma } \\
\hline Yes & 28 & 6.8 \\
\hline No & 254 & 62.1 \\
\hline Unknown & 127 & 31.1 \\
\hline \multicolumn{3}{|l|}{ SBR grading } \\
\hline I & 17 & 4.1 \\
\hline$\|$ & 206 & 50.4 \\
\hline III & 155 & 37.9 \\
\hline Unknown & 31 & 7.6 \\
\hline \multicolumn{3}{|c|}{ Estrogen receptor } \\
\hline positive & 220 & 53.8 \\
\hline negative & 160 & 39.1 \\
\hline Unknown & 29 & 7.1 \\
\hline \multicolumn{3}{|c|}{ Progesterone receptor } \\
\hline Positive & 252 & 61.6 \\
\hline negative & 128 & 31.3 \\
\hline Unknown & 29 & 7.1 \\
\hline \multicolumn{3}{|l|}{ M stage } \\
\hline$\overline{\mathrm{MO}}$ & 356 & 87 \\
\hline M1 & 53 & 13 \\
\hline \multicolumn{3}{|l|}{ TNM Stage } \\
\hline Stage I & 30 & 7.3 \\
\hline Stage II & 140 & 34.2 \\
\hline Stage III & 186 & 45.5 \\
\hline Stage IV & 53 & 13 \\
\hline \multicolumn{3}{|l|}{ HER2 } \\
\hline Positive & 28 & 6.8 \\
\hline negative & 29 & 7.1 \\
\hline unknown & 352 & 86.1 \\
\hline
\end{tabular}

was 32.2 months (range 2- 84.8 months). Table 1 summarizes patient characteristics. The median age at diagnosis was 32 years (range 15-35 years). Twenty-eight patients $(6.8 \%)$ had a family history of breast cancer. BRCA mutation was sought in only a single case and was present. One hundred and twenty one patients (59\%) had nursing antecedents and 129 patients (31.5\%) reported use of oral contraceptives. Three hundred and sixty patients $(88.2 \%)$ had infiltrating ductal carcinoma. Two hundred and six cases (50.4\%) were grade II ScarffBloom-Richardson (SBR), $53.8 \%$ of the cases were estrogen receptors positive, and $61.6 \%$ of the cases were progesterone receptors positive. The Her2 status gene amplification was performed in 57 patients. HER-2 was positive in only 28 patients. Fifty three patients (13\%) had metastatic disease (stage IV) at first diagnosis, 30 patients $(7.3 \%)$ had stage I, 140 patients $(34.2 \%)$ had stage II and the remaining 186 patients $(45.5 \%)$ had stage III.

\section{Treatment and outcome All patients}

Three hundred and fifty six patients (87\%) had local disease at diagnosis and 53 patients (13\%) had metastatic disease. At the end of the study period, 92 patients (22.49\%) died. For all patients, survival at 3 years was $80.6 \%$. Overall survival rate at 3 years was $100 \%, 89.3 \%$, $74.7 \%$, and $57.8 \%$ in stage I, stage II, stage III and stage IV respectively. This difference was statistically significant: Log rank test, $\mathrm{p}<0.001$ (figure 1).

\section{Metastatic disease}

Among 53 patients with metastatic disease, 18 patients (34\%) received surgical treatment. Forty seven patients (88.7\%) received anthracycline-based regimen in the first line metastatic chemotherapy: thirty-seven patients (78.7\%) received AC 60 protocol, six patients (12.8\%)

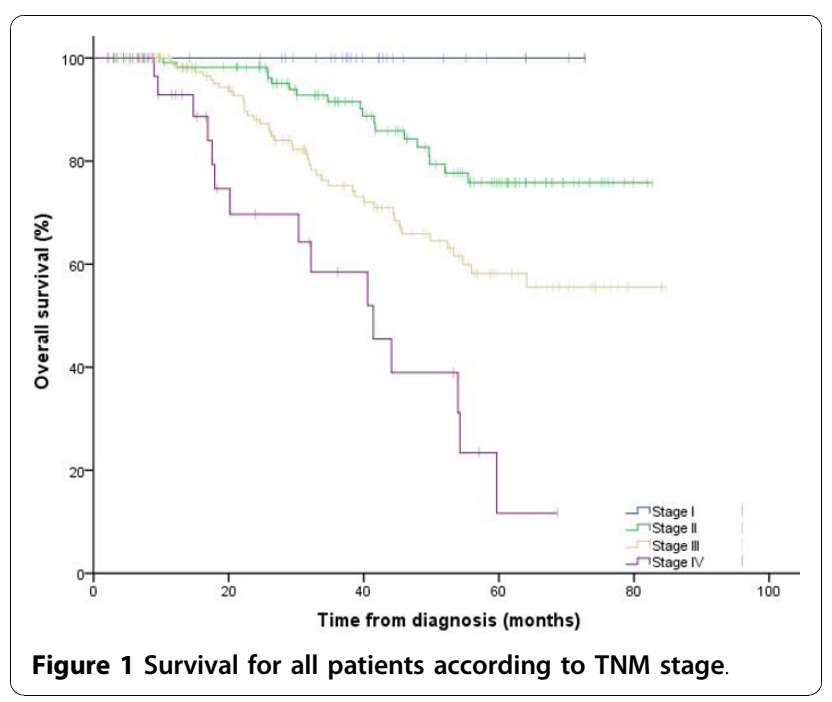


received FEC100 protocol and four patients (8.5\%) received AT protocol. Trastuzumab was not prescribed for any patients with Her 2 positive status (Table 2).

No significant difference in OS at 3 years was seen between the 2 groups of patients how received chemotherapy or not received chemotherapy $(57.7 \%$ vs $50 \%$ respectively; Log rank test: $\mathrm{p}=0.4)($ Table 2$)$.

\section{Non metastatic disease}

From three hundred and fifty six patients how had localized disease, 186 patients (52.2\%) had stage III at diagnosis, 140 patients (39.3\%) with stage II and 30 patients (8.4\%) with stage I (table 3). Positive estrogen receptors status was significantly associated with OS and EFS at 3 years compared to negative estrogen receptors status $(\mathrm{p}=0.001)$. Three hundred and forty seven patients (97.5\%) received surgery in which 80 patients $(23.2 \%)$ had conservative surgery (tumorectomy with axillary lymph nodes). The remaining patients $76.8 \%$ received radical mastectomy with axillary lymph nodes dissection (Patey type mastectomy) (table 3). All patients with local disease, who were operated, received optimal surgery with free histological margins. Patients with advanced tumors or inflammatory breast cancer have received neoadjuvante chemotherapy before surgery. No significant difference in OS and EFS at 3 years was seen in patients how had radical or conserving surgery.(Table 4).

Seventy six patients (21.3\%) received neoadjuvante chemotherapy wich 69 patients $(90.8 \%)$ had Anthracycline based chemotherapy (AC60, FAC50 and FEC100), five patients $(6.6 \%)$ received CMF protocol and two patients (2.6\%) had Anthracycline and taxane protocol (3 cycles of AC60 folowed by 3 cycles of docetaxel). Only two patients had complete response to neoadjuvante chemotherapy. Most patients had a chevalier response 3 and 4 (50 and $39.5 \%$ respectively) (table 3 ).

From 311 patients how received adjuvant chemotherapy, 273 patients (87.8\%) had Anthracycline based chemotherapy (AC60, FAC50 and FEC100), 13 patients (4.2\%) had sequential Anthracycline and docetaxel, 14 patients (4.5\%) received CMF protocol and 11 patients (3.5\%) had docetaxel as adjuvant chemotherapy (table 3). Fifty five patients received previous neoadjuvante chemotherapy.

OS and EFS at 3 years were higher in patients how received adjuvant chemotherapy. (Table 5) Twenty-four patients had HER 2 positive and none of them has received trastuzumab (table 3 ).

According to standard recommendations for localised breast cancer when lymph nodes were affected, 290 patients (81.5\%) received adjuvant radiotherapy (table3). OS and EFS at 3 years were higher in patients how received adjuvant radiotherapy compared to patients how not received adjuvant radiotherapy. The difference was statistically significant (table 4). From 232 patients how had hormone therapy, 230 patients (64.6\%) received tamoxifen in adjuvant setting in the case of positive hormone receptors status and two patients received castration (table 3). OS and EFS at 3 years was statistically significant for patients how received hormone therapy ( 85.9 vs $76.6 \% ; \mathrm{p}=0,001$ and 69.5 vs $48 \% ; \mathrm{p}=0,001$ respectively).

At last follow up, forty four patients (12.4\%) experienced local relapse, ninety two patients $(25.8 \%)$ had metastatic progression and sixty nine patients (19.4\%) died. For all patients with localised disease, OS and EFS at 3 years were 83.2 and $62.5 \%$ respectively.

Table 2 Treatment characteristics and overall survival in patients with metastatic disease

\begin{tabular}{|c|c|c|c|c|c|}
\hline & \multirow[t]{2}{*}{ No. of patients(\%) } & \multirow[t]{2}{*}{ 3-Year OS(\%) } & \multicolumn{2}{|c|}{ Median OS } & \multirow[t]{2}{*}{$\mathbf{p}$} \\
\hline & & & Months & $95 \% \mathrm{Cl}$ & \\
\hline \multicolumn{6}{|l|}{ Surgery } \\
\hline Yes & $18(34)$ & - & - & - & - \\
\hline No & $35(66)$ & - & - & - & \\
\hline \multicolumn{6}{|c|}{ Metastatic First line Chemotherapy } \\
\hline No chemotherapy & $6(11.3)$ & 50 & 20.2 & $0-40.4$ & 0.40 \\
\hline Chemotherapy & $47(88.7)$ & 57.7 & 39 & $30.6-47.3$ & \\
\hline \multicolumn{6}{|c|}{ Chemotherapy protocol } \\
\hline AC 60 & $37(78.7)$ & 57.7 & 41.4 & $21.6-61.2$ & 0.65 \\
\hline FEC 100 & $6(12.8)$ & 33.3 & 35.1 & $30.4-39.8$ & \\
\hline AT & $4(8.5)$ & NR & 39 & NR & \\
\hline \multicolumn{6}{|l|}{ Hormonotherapy } \\
\hline No hormonotherapy & $41(77.4)$ & 58.3 & 39 & $27.1-50.8$ & 0.60 \\
\hline hormonotherapy & $12(22.6)$ & 48.4 & 35.1 & $5.5-64.8$ & \\
\hline
\end{tabular}


Table 3 Treatments and Disease characteristics in patients with local disease

\begin{tabular}{|c|c|c|}
\hline Characteristic & No. of patients & (\%) \\
\hline No. of patients & 356 & 100 \\
\hline \multicolumn{3}{|l|}{ Tumor size } \\
\hline T1 & 48 & 13.5 \\
\hline $\mathrm{T} 2$ & 161 & 45.2 \\
\hline T3 & 71 & 19.9 \\
\hline T4 & 76 & 21.3 \\
\hline \multicolumn{3}{|l|}{ N Statut } \\
\hline No & 107 & 30.1 \\
\hline N1 & 74 & 20.8 \\
\hline N2 & 93 & 26.1 \\
\hline N3 & 82 & 23 \\
\hline \multicolumn{3}{|l|}{ HER 2} \\
\hline HER 2 negative & 23 & 48.9 \\
\hline HER 2 positive & 24 & 51.1 \\
\hline \multicolumn{3}{|l|}{ TNM } \\
\hline Stage I & 30 & 8.4 \\
\hline Stage II & 140 & 39.3 \\
\hline Stage III & 186 & 52.2 \\
\hline \multicolumn{3}{|l|}{ Surgery } \\
\hline Yes & 347 & 97.5 \\
\hline No & 9 & 2.5 \\
\hline \multicolumn{3}{|l|}{ Surgery type } \\
\hline Radical mastectomy & 265 & 76.8 \\
\hline Concerving surgery & 80 & 23.2 \\
\hline \multicolumn{3}{|l|}{ Radiotherapy } \\
\hline Yes & 290 & 81.5 \\
\hline No & 66 & 18.5 \\
\hline \multicolumn{3}{|c|}{ Neoadjuvante chemotherapy } \\
\hline Yes & 76 & 21.3 \\
\hline No & 280 & 78.7 \\
\hline \multicolumn{3}{|c|}{ Neoadjuvante chemotherapy type } \\
\hline Anthracycline & 69 & 90.8 \\
\hline CMF & 5 & 6.6 \\
\hline AC60/TAXANE & 2 & 2.6 \\
\hline \multicolumn{3}{|c|}{ Histological response to neoadjuvante chemotherapy } \\
\hline Chevalier 1 & 2 & 2.6 \\
\hline Chevalier 2 & 6 & 7.9 \\
\hline Chevalier 3 & 38 & 50 \\
\hline Chevalier 4 & 30 & 39.5 \\
\hline \multicolumn{3}{|c|}{ Adjuvant chemotherapy } \\
\hline Yes & 311 & 87.4 \\
\hline No & 45 & 12.6 \\
\hline \multicolumn{3}{|c|}{ Adjuvant hormonotherapy } \\
\hline Yes & 232 & 64.6 \\
\hline No & 124 & 35.4 \\
\hline
\end{tabular}

Table 4 OS and EFS in patients35 years and younger with local breast cancer

\begin{tabular}{|c|c|c|c|c|c|}
\hline & $\begin{array}{c}\text { No. of } \\
\text { patients }\end{array}$ & $\begin{array}{c}\text { 3-Year OS } \\
\text { (\%) }\end{array}$ & $\mathbf{P}$ & $\begin{array}{c}\text { 3-Year EFS } \\
(\%)\end{array}$ & $\mathbf{P}$ \\
\hline Stage I-III & 356 & 83.2 & - & 62.5 & - \\
\hline \multicolumn{6}{|l|}{ Tumor size } \\
\hline T1 & 48 & 89.3 & \multirow[t]{4}{*}{0.76} & 75.9 & \multirow[t]{4}{*}{0.32} \\
\hline $\mathrm{T} 2$ & 161 & 85.5 & & 65 & \\
\hline T3 & 71 & 79.5 & & 56 & \\
\hline $\mathrm{T4}$ & 76 & 77.5 & & 55.2 & \\
\hline \multicolumn{6}{|c|}{ SBR grading } \\
\hline SBR I & 14 & 100 & \multirow[t]{3}{*}{0.20} & 67.5 & \multirow[t]{3}{*}{-0.45} \\
\hline SBR ॥ & 176 & 84.9 & & 63.2 & \\
\hline SBR III & 137 & 78 & & 58.8 & \\
\hline \multicolumn{6}{|l|}{ ER } \\
\hline ER positive & 179 & 94.4 & \multirow[t]{2}{*}{0.001} & 75.8 & \multirow[t]{2}{*}{0.001} \\
\hline ER negative & 149 & 70.2 & & 50.6 & \\
\hline
\end{tabular}

\section{$\mathrm{N}$ stage}

\begin{tabular}{|c|c|c|c|c|c|}
\hline No & 107 & 79.4 & 0.32 & 59.8 & 0.20 \\
\hline $\mathrm{N}+$ & 249 & 84.8 & & 63.6 & \\
\hline \multicolumn{6}{|l|}{ Surgery type } \\
\hline $\begin{array}{l}\text { Radical } \\
\text { mastectomy }\end{array}$ & 265 & 82.3 & 0.17 & 61.9 & 0.059 \\
\hline $\begin{array}{l}\text { Conserving } \\
\text { surgery }\end{array}$ & 80 & 87.7 & & 70.4 & \\
\hline
\end{tabular}

\begin{tabular}{|c|c|c|c|c|c|}
\hline \multicolumn{6}{|c|}{ Adjuvant radiotherapy } \\
\hline Yes & 290 & 85.2 & 0.002 & 65.7 & 0.001 \\
\hline No & 66 & 70.2 & & 48.6 & \\
\hline
\end{tabular}

Adjuvant chemotherapy

\begin{tabular}{|c|c|c|c|c|c|}
\hline No & 45 & 65.4 & \multirow[t]{2}{*}{0.04} & 44.7 & \multirow[t]{2}{*}{0.001} \\
\hline Yes & 311 & 85 & & 64.9 & \\
\hline \multicolumn{6}{|c|}{ Hormonotherapy } \\
\hline Yes & 232 & 85.9 & \multirow[t]{2}{*}{0.001} & 69.5 & \multirow[t]{2}{*}{0.001} \\
\hline No & 124 & 76.6 & & 48 & \\
\hline
\end{tabular}

Stage

\begin{tabular}{|c|c|c|c|c|}
\hline I & 30 & 100 & 0.001 & 93 \\
\hline$\|$ & 140 & 89.4 & & 76.3 \\
\hline |II & 186 & 74.7 & & 46.8 \\
\hline
\end{tabular}

ER. estrogen receptors; N. lymph node; Cl. confidence interval; OS. overall survival; EFS. event free survival.

\section{Univariate and multivariate Cox regression analysis}

Factors influencing survival Univariate analysis showed that the factors significantly influencing OS were the negative ER status, advanced tumoral stage, absence of adjuvant chemotherapy and adjuvant endocrine therapy for patients with positive ER, and absence of adjuvant radiotherapy (table 5). Multivariate analysis showed that only negative ER was significantly associated with poorer overall survival. $(\mathrm{HR}=2.54,95 \% \mathrm{CI}=1.32-4.90, \mathrm{p}<$ 0.005) (table 5). 
Table 5 Cox Proportional Hazards Model of Overall Survival for patients with local disease

\begin{tabular}{|c|c|c|c|c|c|c|}
\hline & \multicolumn{2}{|c|}{ Univariate analysis } & \multirow[b]{2}{*}{$P$-value } & \multicolumn{2}{|c|}{ Multivariate analysis } & \multirow[b]{2}{*}{$P$-value } \\
\hline & Hazard ratio & $95 \% \mathrm{Cl}$ & & Hazard ratio & $95 \% \mathrm{Cl}$ & \\
\hline \multicolumn{7}{|l|}{ Family history } \\
\hline No & 1.00 & & & 1.00 & & \\
\hline Yes & 1.10 & $0.47-2.58$ & 0.83 & 1.12 & $0.44-2.88$ & 0.81 \\
\hline \multicolumn{7}{|l|}{ SBR grading } \\
\hline SBR I & 1.00 & & & 1.00 & & \\
\hline SBR II & 2.01 & $0.27-14.6$ & 0.49 & 1.26 & $0.15-10.9$ & 0.83 \\
\hline SBR III & 2.55 & $0.35-18.69$ & 0.36 & 1.03 & $0.12-8.76$ & 0.97 \\
\hline \multicolumn{7}{|l|}{ Tumor size } \\
\hline $\mathrm{T} 1$ & 1.00 & & & 1.00 & & \\
\hline $\mathrm{T} 2$ & 1.54 & $0.65-3.66$ & 0.33 & 2.25 & $0.50-10.19$ & 0.30 \\
\hline T3 & 1.55 & $0.59-4.1$ & 0.38 & 2.50 & $0.47-13.42$ & 0.29 \\
\hline $\mathrm{T} 4$ & 1.64 & $0.64-4.23$ & 0.31 & 2.34 & $0.44-12.44$ & 0.32 \\
\hline \multicolumn{7}{|l|}{$\mathrm{N}$ statut } \\
\hline $\mathrm{N}-$ & 1.00 & & & 1.00 & & \\
\hline $\mathrm{N}+$ & 0.78 & $0.47-1.28$ & 0.32 & 0.62 & $0.29-1.30$ & 0.20 \\
\hline \multicolumn{7}{|l|}{ Stage } \\
\hline I & 1.00 & & & 1.00 & & \\
\hline$\|$ & 2.10 & $0.53-8.37$ & 0.29 & 1.43 & $0.24-8.57$ & 0.69 \\
\hline III & 4.24 & $1.1-16.28$ & 0.03 & 3.56 & $0.65-19.57$ & 0.14 \\
\hline \multicolumn{7}{|l|}{ ER } \\
\hline ER positive & 1.00 & & & 1.00 & & \\
\hline ER negative & 2.25 & $1.37-3.72$ & 0.001 & 2.54 & $1.32-4.90$ & 0.005 \\
\hline \multicolumn{7}{|c|}{ Adjuvant chemotherapy } \\
\hline No & 1.00 & & & 1.00 & & \\
\hline Yes & 0.49 & $0.24-0.99$ & 0.04 & 0.74 & $0.18-3.05$ & 0.68 \\
\hline \multicolumn{7}{|c|}{ Adjuvant hormonotherapy } \\
\hline No & 1.00 & & & 1.00 & & \\
\hline Yes & 0.45 & $0.28-0.73$ & 0.001 & 0.89 & $0.45-1.77$ & 0.74 \\
\hline \multicolumn{7}{|l|}{ Surgery type } \\
\hline Radical mastectomy & 1.00 & & & 1.00 & & \\
\hline Conserving surgery & 0.64 & $0.34-1.23$ & 0.17 & 0.50 & $0.19-1.18$ & 0.11 \\
\hline \multicolumn{7}{|c|}{ Adjuvant radiotherapy } \\
\hline No & 1.00 & & & 1.00 & & \\
\hline Yes & 0.42 & $0.24-0.74$ & 0.003 & 0.74 & $0.25-2.23$ & 0.60 \\
\hline
\end{tabular}

1. Reference; ER. estrogen receptors; N. lymph node; Cl. confidence interval; OS. overall survival; EFS. Events free survival.

Factors influencing event free survival Univariate analysis indicated that advanced tumoral stage, negative ER, absence of adjuvant chemotherapy, radiotherapy, and hormone therapy, and conservative surgery were all risk factors of poorer event free survival (table 6), however multivariate analysis showed that negative ER status and advanced stage III disease were the only factors associated with poorer event free survival (table 6).

\section{Discussion}

This work conducted at the national institute of oncology in morocco analyzed the epidemiological, clinical, therapeutic and prognostic characteristics of breast cancer in women aged 35 years or less and the relationship between these characteristics and outcome (EFS and OS).

Of 5309 patients diagnosed with breast cancer between 2003 and 2007, 8\% of the cases were aged 35 
Table 6 Cox Proportional Hazards Model of Event free survival for patients with local disease

\begin{tabular}{|c|c|c|c|c|c|c|}
\hline & \multicolumn{2}{|c|}{ Univariate analysis } & \multirow[b]{2}{*}{$P$-value } & \multicolumn{2}{|c|}{ Multivariate analysis } & \multirow[b]{2}{*}{$P$-value } \\
\hline & Hazard ratio & $95 \% \mathrm{Cl}$ & & Hazard ratio & $95 \% \mathrm{Cl}$ & \\
\hline \multicolumn{7}{|l|}{ Family history } \\
\hline No & 1.00 & & & 1.00 & & \\
\hline Yes & 1.25 & $0.66-2.34$ & 0.49 & 0.86 & $0.41-1.84$ & 0.71 \\
\hline \multicolumn{7}{|l|}{ SBR grading } \\
\hline SBR I & 1.00 & & & 1.00 & & \\
\hline$\overline{S B R} \|$ & 0.66 & $0.26-1.64$ & 0.36 & 0.62 & $0.17-2.25$ & 0.47 \\
\hline SBR III & 0.79 & $0.32-1.99$ & 0.63 & 0.69 & $1.19-2.47$ & 0.57 \\
\hline \multicolumn{7}{|l|}{ Tumor size } \\
\hline $\mathrm{T1}$ & 1.00 & & & 1.00 & & \\
\hline $\mathrm{T} 2$ & 1.26 & $0.69-2.30$ & 0.45 & 1.27 & $0.51-3.17$ & 0.61 \\
\hline $\mathrm{T} 3$ & 1.68 & $0.87-3.23$ & 0.12 & 1.10 & $0.35-3.14$ & 0.93 \\
\hline $\mathrm{T4}$ & 1.56 & $0.82-2.98$ & 0.17 & 1.40 & $0.48-3.81$ & 0.57 \\
\hline \multicolumn{7}{|l|}{$\mathrm{N}$ statut } \\
\hline $\mathrm{N}-$ & 1.00 & & & 1.00 & & \\
\hline $\mathrm{N}+$ & 0.79 & $0.55-1.13$ & 0.20 & 0.74 & $0.42-1.30$ & 0.29 \\
\hline \multicolumn{7}{|l|}{ Stage } \\
\hline I & 1.00 & & & 1.00 & & \\
\hline$\|$ & 4.33 & $1.04-17.9$ & 0.04 & 2.03 & $0.57-7.25$ & 0.27 \\
\hline III & 10.96 & $2.70-44.5$ & 0.01 & 5.35 & $1.60-17.84$ & 0.01 \\
\hline \multicolumn{7}{|l|}{ ER } \\
\hline ER positive & 1.00 & & & 1.00 & & \\
\hline ER negative & 1.83 & $1.26-2.64$ & 0.01 & 1.63 & $1.00-2.67$ & 0.05 \\
\hline \multicolumn{7}{|c|}{ Adjuvant chemotherapy } \\
\hline No & 1.00 & & & 1.00 & & \\
\hline Yes & 0.45 & $0.28-0.73$ & 0.001 & 0.90 & $0.33-2.52$ & 0.85 \\
\hline \multicolumn{7}{|c|}{ Adjuvant hormonotherapy } \\
\hline No & 1.00 & & & 1.00 & & \\
\hline Yes & 0.43 & $0.30-0.60$ & 0.001 & 0.73 & $0.43-1.23$ & 0.24 \\
\hline \multicolumn{7}{|l|}{ Surgery type } \\
\hline Radical mastectomy & 1.00 & & & 1.00 & & \\
\hline Conserving surgery & 0.63 & $0.40-1.01$ & 0.05 & 0.74 & $0.42-1.13$ & 0.30 \\
\hline \multicolumn{7}{|c|}{ Adjuvant radiotherapy } \\
\hline No & 1.00 & & & 1.00 & & \\
\hline Yes & 0.45 & $0.30-0.68$ & 0.001 & 0.82 & $0.35-1.19$ & 0.64 \\
\hline
\end{tabular}

years or younger. The higher age adjusted incidence could be partially attributed to the higher proportion of young women in the general population. In the US, the women aged 35 years or younger represent only $2.7 \%$ of new cases [10]. In Asian series, this number varies between $10 \%$ in developed and up to $24 \%$ in developing countries [11].

This study found less than $10 \%$ of family history of breast cancer and no evidence for a relationship between family history of breast cancer and survival. However, family history of breast cancer is an important indicator of risk in young women. In a Swedish population-based study of 262 women with breast cancer aged 40 years or younger, $48 \%$ of patients had a family history of breast or ovarian cancer [12].

The reports studied the impact of age and others prognostic factors showed that high tumor grade represents an individual prognostic factor in younger and older premenopausal patients [13]. In addition, several studies published after 1995 reported that the unfavorable impact of young age on survival was present only in patients who did not receive chemotherapy [14]. 
However, Colleoni et al. [15], and Kothari et al. [16], showed that the outcome of patients aged $\leq 35$ years was less favorable than those aged $>35$ years. In fact, the outcome of young women with localized disease included in the present study is less favorable than that of patients having localized disease followed in the same Institute and included in a previous study [17]. Also, in this work, we found that women were more likely to receive adjuvant chemotherapy and they had a better survival, however high tumor grade was not influenced survival.

Our findings revealed that negative ER status was associated with lower survival and event free survival in univariate and multivariate analysis. Curigliano [18] and Aebi [19] noted that the positive ER status in young patients seems to have a different prognostic value. Data suggest that very young women with endocrine-responsive tumors had a statistically significant higher risk of disease recurrence than older premenopausal patients. In contrast, results in younger and older premenopausal patients were similar in the case of the ER positive status. However, Ana M. Gonzalez-Angulo[20] et all found that Hormone receptor negativity was associated with shorter RFS and OS. This result was consistent with our finding.

Breast cancer in young women is frequently diagnosed at advanced stage [21]. Consequently, the majority of patients received radical mastectomy. In addition, a positive family history or the presence of a BRCA $1 / 2$ gene mutation will also influence decisions for mastectomy rather than breast conservation [3]. Until now, there is still no final conclusion about whether age is a risk factor of local recurrence in breast conserved cases, and there are still inconsistent findings in previous clinical studies. But it was reported in most studies that among those who received breast conserving surgery, young patients had a higher local recurrence rate. [22-28] Over $20 \%$ of our patients with local disease received conservative surgery. These patients had lower event-free survival than patients who received radical mastectomy. The difference tended towards significance.

The updated Early Breast Cancer Clinical Trialists' meta-analysis have shown beneficial effects of adjuvant tamoxifen in younger women [29], as well as the findings from the International Breast Cancer Study Group (Trial 13-93) [30]. In our series, the patients with localised disease and estrogen receptor positive status, how had received the hormonal treatment had better OS and EFS.

However our retrospective study showed several limitations because it implicated potential bias in the choice of treatment. In addition, the lake of cytogenetic investigation of BRCA gene mutation due to the low socioeconomic level of these patients and the lake of Her-2 gene amplification test in the majority of our patients are 2 major limitations.

\section{Conclusion}

In Morocco, the incidence of breast cancer in young women aged $\leq 35$ is higher compared than that in developed countries. In this subgroup of patients, the invasive breast cancer has more aggressive behaviors. In addition, the ER negative status was associated with lower EFS and OS. Further research program and clinical trials were needed in young Moroccan breast cancer women improve their management and their outcome.

\section{List of abriviations}

Cl: Confidence interval; ER: Estrogen receptor; EFS: Event free survival; HR: Hazard ratio; IHC: Immunohistochemistry; N: lymph nodes; OS: Overall surviaval; SD: Standard deviation.

\section{Acknowledgements}

I wish to thank Dr Erraki Mohamed from the epidemiology unit at National Institute of Oncology and his team. I thank also the Laboratory of Biostatistics, Epidemiology and Clinical Research team in Rabat and Pr Mohammed Ismaili from the Department of Microbiology, Moulay Ismail University, Meknes, Morocco.

\section{Author details}

${ }^{1}$ Department of medical oncology, National Institute of Oncology, Rabat, Morocco. ${ }^{2}$ Department of radiotherapy, National Institute of Oncology, Rabat, Morocco. ${ }^{3}$ Department of Medical Oncology, Hassan II University Hospital, Fez, Morocco. ${ }^{4}$ Laboratory of Biostatistics, Epidemiology and Clinical Research, Rabat, Morocco. ${ }^{5}$ Epidemiology Unit, National institute of oncology, Rabat, Morocco.

\section{Authors' contributions}

HA has conceived the study, exploited data, coordinated, drafted and wrote the manuscript. IL, FEM and $\mathbf{N I}$ participated in the design and data exploitation and its input. RA, NI, RR and $\mathbf{M A T}$ have performed the statistical analysis. RA, $\mathbf{N I}$ and $\mathbf{M A T}$ revised the manuscript. HM, OEM, NB, RA and $\mathbf{H E}$ participated in the design of this study, and revised the manuscript. All authors read and approved the final manuscript.

\section{Competing interests}

The authors declare that they have no competing interests.

Received: 27 May 2010 Accepted: 8 November 2010

Published: 8 November 2010

\section{References}

1. Merrill RM, Capocaccia R, Feuer EJ, Mariotto A: Cancer prevalence estimates based on tumour registry data in the Surveillance, Epidemiology, and End Results (SEER) program. Int J Epidemiol 2000, 29:197-207.

2. Bland KI, Menck HR, Scott-Conner CE, Morrow M, Winchester DJ, Winchester DP: The National Cancer Data Base 10-year survey of breast carcinoma treatment at hospitals in the United States. Cancer 1998, 83:1262-1273.

3. Axelrod D, Smith J, Kornreich D, Grinstead E, Singh B, Cangiarella J, Guth AA: Breast cancer in young women. J Am Coll Surg 2008, 206(3):1193-203, Epub 2008 Apr 24.

4. Adami HO, Malker B, Holmberg L, Persson I, Stone B: The relation between survival and age at diagnosis in breast cancer. N Engl J Med 1986, 315:559-563.

5. Chung M, Chang HR, Bland KI, Wanebo HJ: Younger women with breast carcinoma have a poorer prognosis than older women. Cancer 1996, 77:97-103. 
6. Walker RA, Lees E, Webb MB, Dearing SJ: Breast carcinomas occurring in young women ( $\leq 35$ years) are different. Br J Cancer 1996, 74:1796-1800.

7. Xiong QH, Valero V, Kau SW, et al: Female patients with breast carcinoma age 30 years and younger have a poor prognosis-The M. D. Anderson Cancer Center Experience. Cancer 2001, 92:2523-2528.

8. Thuerlimann B: International consensus meeting on the treatment of primary breast cancer 2001, St. Gallen, Switzerland. Breast Cancer 2001, 8:294-297.

9. Chevallier B, Chollet P, Merrouche $Y$, Roche H, Fumoleau P, Kerbrat $P$, Genot JY, Fargeot P, Olivier JP, Fizames C, Clavelt M, Yver A, Chabernaud VC: Lenograstim prevents morbidity from intensive induction chemotherapy in the treatment of inflammatory breast cancer. J Clin Oncol 1995, 13:1564-71.

10. Winchester DP, Osteen RT, Menck HR: The National Cancer Data Base report on breast carcinoma characteristics and outcome in relation to age. Cancer 1996, 78(8):1838-1843.

11. Agarwal G, Pradeep PV, Aggarwal V, et al: Spectrum of breast cancer in Asian women. World J Surg 2007, 31:1031-1040.

12. Loman $\mathrm{N}$, Johannsson $\mathrm{O}$, Kristoffersson $\mathrm{U}$, et al: Family history of breast and ovarian cancers and BRCA1and BRCA2 Mutations in a populationbased series of early-onset breast cancer. J Nat Cancer Inst 2001, 93:1215-1223.

13. Nemoto T, Vana J, Bedwani RN: Management and survival of female breast cancer: results of a national survey by the American College of Surgeons. Cancer 1980, 45:2917-2924.

14. Rapiti E, Fioretta G, Verkooijen HM, et al: Survival of young and older breast cancer patients in Geneva from 1990 to 2001. Eur J Cancer 2005, 41:1446-1452.

15. Colleoni M, Rotmenz N, et al: Very young women ( $<35$ years) with operable breast cancer: features of disease at presentation. [J] Ann Oncol 2002, 13(2):273-279.

16. Kothari AS, Beechey-Newman N, D'Arrigo C, et al: Breast carcinoma in women age 25 years or less. [J] Cancer 2002, 94(3):606-614.

17. Ismaili N, Mellas N, Masbah O, Elmaijaoui S, Arifi S, Bekkouch I, Ahid S, Bazid Z, Tazi MA, Erraki A, El Mesbahi O, Benjaafar N, El Gueddari Bel K, Ismaili M, Afair S, Errihani H: Concurrent chemoradiotherapy in adjuvant treatment of breast cancer. Radiat Oncol 2009, 7:4:12.

18. Curigliano $G$, Rigo $R$, Colleoni $M$, et al: Adjuvant therapy for very young women with breast cancer: response according to biologic and endocrine features. Clin Breast Cancer 2004, 5:125-130.

19. Aebi S, Gelber S, Castiglione-Gertsch M, et al: Is chemotherapy alone adequate for young women with oestrogen-receptor- positive breast cancer? Lancet 2000, 355:1869-1874.

20. Gonzalez-Angulo AM, Broglio K, Kau SW, Eralp Y, Erlichman J, Valero V Theriault R, Booser D, Buzdar AU, Hortobagyi GN, Arun B: Women age < or $=35$ years with primary breast carcinoma: disease features at presentation. Cancer 2005, 103(12):2466-72.

21. Wapnir IL, Anderson SJ: Prognosis after ipsilateral breast cancer recurrence and locoregional recurrences in five National Surgical Adjuvant Breast and Bowel Project node-positive adjuvant breast cancer trials. J Clin Oncol 2006, 24:2028-2037.

22. Vielling C, Collecte L, Fourquet A, et al: Can patient-, treatement- and pathology-related caracteristics explain the high local recurrence rate following breast-conserving therapy in young patients? [J] Eur J Cancer 2003, 39(7):932-944.

23. Zhou P, Recht A: Young age and outcome for women with early-stage invasive breast carcinoma. [J] Cancer 2004, 101:1264-1274

24. Voogd AC, Nielsen M, Peterse $\mathrm{J}$, et al: Differences in risk factors for local and distant recurrence after breast-conserving thearapy or mastectomy for stage I and II breast cancer: pooled results of two large European randomized trials. [J] J Clin Oncol 2001, 19(6):1688-1697.

25. Jobsen JJ, van der Palen J, Ong F, et al: The value of a positive margin for invasive carcinoma in breast-conservative treatment in relation to local recurrence is limited to young women only. [J] Int I Radiat Oncol Biol Phys 2003, 57(3):724-731.

26. Neuschatz AC, Dipetrillo T, Safall H, et al: Long-term follow-up of a prospective policy of margin-directed radiation dose escalation in breast-conserving therapy. [J] Cancer 2003, 97(1):30-39.

27. Elkhuizen $\mathrm{PH}$, van Slooten $\mathrm{HJ}$, Clahsen $\mathrm{PC}$, et al: High local recurrence risk after breast-conserving therapy in node-negative premenopausal breast cancer patients is greatly reduced by one course of perioperative chemotherapy: a European Organization for research and treatment of Cancer Breast Cancer Cooperative Group Study. [J] J Clin Oncol 2000, 18(5):1075-1083

28. Veronesi U, Cascinelli N, Mariani L, et al: Twenty-year follow-up of a randomized study comparing breast-conserving surgery with radical mastectomy for early breast cancer. [J] N Engl J Med 2002, 347(16):1227-1232.

29. Early Breast Cancer Trialist's Collaborative Group (EBCTCG): Effects of chemotherapy and hormonal therapy for early breast cancer on recurrence and 15-year survival: an overview of the randomised trials. Lancet 2005, 365:1687-1717.

30. Colleoni M, Gelber S, Goldhirsch A, et al: Tamoxifen after adjuvant chemotherapy for premenopausal women with lymph node-positive breast cancer: International Breast Cancer Study Group Trial 13-93. I Clin Oncol 2006, 24:1332-1341.

doi:10.1186/1756-0500-3-286

Cite this article as: Abahssain et al: Breast cancer in moroccan young women: a retrospective study. BMC Research Notes 2010 3:286.

\section{Submit your next manuscript to BioMed Central and take full advantage of:}

- Convenient online submission

- Thorough peer review

- No space constraints or color figure charges

- Immediate publication on acceptance

- Inclusion in PubMed, CAS, Scopus and Google Scholar

- Research which is freely available for redistribution 